\title{
Analysis on the Relationship Between Different Body and Mind States and Sleep Quality
}

\author{
Leyao Ma
}

\author{
Annie Wright School, Tacoma Washington State, 48407, USA \\ *Corresponding author.Email: shilishuang@cas-harbour.org
}

\begin{abstract}
The definition of sleep quality is not consistent, but several indicators, including sleep efficiency, the total wake time, total sleep time, the latency of sleep onset, the degree of fragmentation, and even sleep disruptive events have been used to measure it. However, while the physiological aspects of sleep quality on the human body have received much scholarly interest, there is lesser interest into the emotive factors that influence sleep quality. This research reviews the effects of stress, tiredness and relaxation on sleep quality, and in doing so, hopes to rekindle research interest into this area of research. According to the research results, stress and fatigue or tiredness both have an adverse cyclic relationship with sleep quality However, relaxation has the complete opposite impact on sleep quality as stress, as these two are naturally antagonistic body responses. There are many explainations on these phenomena. However, it still requires the researchers to do further research on this area. Regardless, additional research is required that explores further than correlative relationships between sleep quality and influencing factors using subjective data.
\end{abstract}

Keywords: Sleep quality, stress, tiredness, fatigue, relaxation, meditation

\section{INTRODUCTION}

Sleep quality is a term that has been widely used in the scholarly and medical discussions surrounding sleep and sleep therapies. However, Krystal and Edinger indicated that for the longest time, there has not been an established and consistent definition for the term [3]. However, there is relative consensus on the various measures that are used to refer to sleep quality, including sleep efficiency, the total wake time, total sleep time, the latency of sleep onset, the degree of fragmentation, and even sleep disruptive events [3], [7]. These are the most popular measurements used in popularly-implemented scales like the Pittsburgh Sleep Quality Index (PSQI), and therefore, sleep quality has been broadly defined in accordance to good scores on these measurements.

There has also been significant interest into the various factors that influence sleep and sleep quality. In fact, sleep quality in several developing and developed nations is increasingly becoming a public health problem with several lifestyle and medical conditions being indicated as having a cyclic influence on sleep quality [8]. Mental health conditions like anxiety and depression have been identified as contributing to poor sleep quality and increasing sleep deprivation, which in turn worsens the psychological health of an individual; resulting in a vicious, unhealthy cycle [8]. Therefore, sleep quality is essential to supporting mental health, and also physical health, and is thus essential to ensuring overall quality of life.

Therefore, despite the vagueness in the definition of sleep quality, it is broadly understood how good sleep quality impacts the body. However, the literature is significantly less clear on the various factors that influence sleep quality. Some physiological factors, like medical conditions that result in chronic pain or bodily discomfort or changes in the body and brain, external environmental factors like loud sounds and bright lights, and even ingested contents like food, drink and medication have been widely-researched [5], [7], [8]. The impact of body and mind states on sleep quality fails to secure significant scholarly interest. Therefore, this research is aimed at 
discussing how body and mind states affect sleep quality, with the hope that it can raise more research interest into the field of study.

\section{STRESS AND SLEEP QUALITY}

While the term stress has various negative connotations, it is in essence a very significant evolutionary response in both human beings and animals. Stress is a psychological response that helps human beings react to important and dangerous situations [11]. In human beings, the presence of stress signals the automatic nervous system (ANS) to release the necessary hormones responsible for the appropriate physiological and psychological response; often adrenaline and cortisol [4]. These two hormones, cortisol and adrenaline, are responsible for increasing the heart rate, which in turn ensures the efficient circulation of blood to the more vital organs and muscles, and hence primes the body for immediate and strenuous action if required. This reaction is more popularly called the 'fightor-flight' response, and it is often vital for human beings' and animals' survival in their respective environments as it dictates their reaction in the presence of imminent danger, threats to their survival, or other stressors.

\subsection{Impact of stress on sleep}

However, stress has a negative influence on human beings. One common impact of stress on the body is diminished sleep quality [11]. There is a correlative relationship between stress and sleep quality, which results in what is popularly termed as the stress-sleep cycle [11]. Higher stress levels result into poorer sleep quality, which in turn further worsens the stress levels resulting into a highly vicious cycle. For instance, in a survey conducted by the American Psychologists Association, adults who reported lower stress levels also indicated that they slept more self-measured hours a night, at an average of 7.1 hours, versus individuals with higher stress levels reported to sleep 6.2 hours a night. $33 \%$ of people with lower stress also reported better quality of sleep, as compared to only $8 \%$ of individuals with higher stress levels [11]. Therefore, the level of stress was negatively correlated to the quality and quantity of sleep; with higher stress levels resulting into increasingly diminished quality and duration of sleep.

In human beings, stressors can be varied and contextual; sometimes not even posing a real threat to the survival of the individual. An example of such stressors may include relationship difficulties and work issues. While it is perfectly normal to feel occasionally stressed, the chronic onset of stress may cause the ANS to remain in an overlyheightened state of stimulation for an extended period of time [4]. Being in such a state of chronic stress may have significant adverse impacts on the psychological and physiological health of an individual.

\subsection{Explanations of this phenomenon}

One common impact of stress on the body is diminished sleep quality [11]. There is a correlative relationship between stress and sleep quality, which results in what is popularly termed as the stress-sleep cycle [10]. Higher stress levels result into poorer sleep quality, which in turn further worsens the stress levels resulting into a highly vicious cycle. For instance, in a survey conducted by the American Psychologists Association, adults who reported lower stress levels also indicated that they slept more selfmeasured hours a night, at an average of 7.1 hours, versus individuals with higher stress levels reported to sleep 6.2 hours a night. $33 \%$ of people with lower stress also reported better quality of sleep, as compared to only $8 \%$ of individuals with higher stress levels [11].

Therefore, the level of stress was negatively correlated to the quality and quantity of sleep; with higher stress levels resulting into increasingly diminished quality and duration of sleep.

Many reasons may explain why stress has a negative impact on sleep. One of them is that being in a sustained level of heightened stress results in higher alertness, and therefore delays the onset of sleep. Additionally, stress may cause anxious and rapid thoughts to occur throughout the night, resulting in sleep deprivation [2].

In turn, sleep deprivation may result in additional stress, which creates a vicious cycle. The prevalence of stress is increasing significantly in both developing and developed countries. For instance, the National Sleep Foundation found that an approximate $43 \%$ of people from ages 13 to 64 reported lying awake at night as a result of stress over the past month [6]. Therefore, ways to reduce stress levels, especially before bedtime, like mindful meditation, exercise and other lifestyle changes to improve sleep quality, and offset additional stress are very important to people

\section{TIREDNESS AND SLEEP QUALITY}

\subsection{Impact of tiredness on sleep}

Tiredness is defined as a symptom, and is similarly described as fatigue. Tiredness is experienced by an individual deep within the skeletomuscular structure, and is often characterized by several traits including sore eyes, heavy limbs and head, sensitivities to noise, cold, general body weakness and a loss of interest [2]. While it may sound counterintuitive, tiredness may not always improve 
a person's ability to fall asleep, and also may not resolve in sleep. While the effect of fatigue, and the levels of fatigue, on sleep quality is not wholly understood [2], there is a fairly supported hypothesis that fatigue influences key physiological mediators of sleep quality, and severe fatigue also results in the higher prevalence of sleep disorders.

\subsection{Explanations of this phenomenon}

Fortier-Brochu et al. also posit that, in studies conducted among a variety of sleepers, a correlative relationship was identified between fatigue and several measures of sleep quality, including time spent awake, sleep duration and sleep efficacy [1].

However, they also found that most of the studies on the impact of fatigue on sleep quality followed a correlative design, which only tests the linear relationship between the two variables; fatigue and sleep quality [2]. Therefore, despite clearly indicating that fatigue was negatively correlated with sleep quality; where higher levels of fatigue would result in worse sleep quality, there is the need for additional research into the different relationship patterns between tiredness and sleep quality. Such studies may bring a better understanding of the mechanisms surrounding sleep quality, and result to more effective sleep therapy interventions.

\section{RELAXATION AND SLEEP QUALITY}

From a psychological standpoint, relaxation is defined as a living being's emotive state that is characterized by low tension, the absence of arousal, and particularly from adverse sources like fear, anxiety and/or anger [9].

When the body is in a relaxed state, the body's autogenic indicators include a slow heartbeat, resulting in lower blood pressure, calm breathing, coolness, and warmth in the abdominal region and the limbs [9]. These indicators are antagonistic to the body's stress response, and thereby signaling that there is no imminent threat to the survival or welfare of the individual.

The relaxation response is an essential step in improving sleep quality. As the body's naturally antagonistic response to stress, relaxation results from the alleviation of stress, and therefore negates the adverse impacts of stress on sleep quality. This is supported by Rusch et al. who indicated that fostering the body's natural relaxation response is critical to helping improve overall sleep quality [9]. For instance, over $62 \%$ of individuals outlined that relaxation techniques, like listening to music or mindful meditation helped improve their quality of sleep. Of these individuals, over 50\% indicated that simple techniques like low-tempo music helped with relaxation, and consequently, sleep using the Pittsburg Sleep Quality Index [1].

Relaxation reduces the release of the body's stress hormones; adrenaline and cortisol, and therefore slows down the heart rate and breathing, allowing the body and the mind to calm down [9]. This effect can be achieved through purposeful stress-relief and relaxation techniques, including mindful meditation, yoga and tai chi. As shown in figure 1, a study by Rebar et al. (2018) found that individuals on the off-shift, and whose live-in partners were also on an off-shift had better sleep quality; indicative that relaxing had a positive correlation to sleep quality [12].

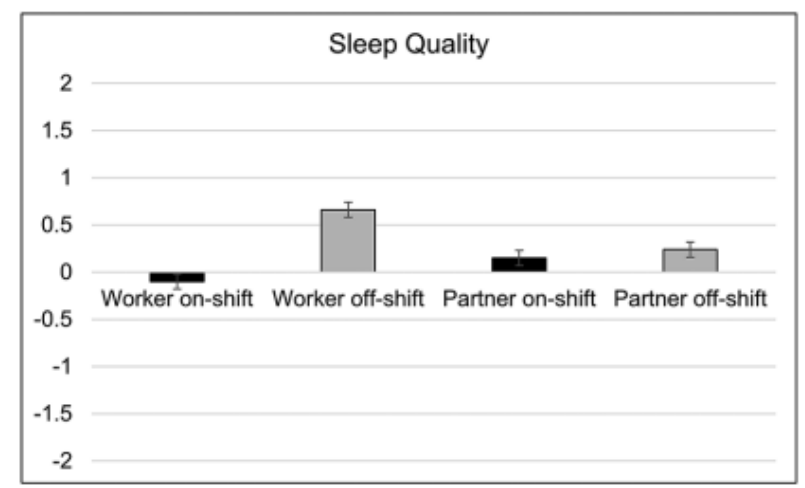

Figure 1. Research result of Rebar et al.(2018)

\section{CONCLUSION}

Sleep quality and stress are closely interlinked, and stress adversely affects sleep quality, which results in a cyclic impact where sleep deprivation in turn elevates stress levels. Therefore, heightened stress levels may lead to adverse psychological and physiological impacts. On the other hand, there is a correlative two-way relationship between tiredness/fatigue and sleep quality. Poor-quality of sleep results in reduced rest and rejuvenation, and therefore worsens tiredness levels, with significant adverse impacts on an individual's mental and physical health.

However, fatigue also diminishes various measures of sleep quality, including time spent awake, sleep duration, and sleep efficiency. Therefore, the relationship between tiredness and sleep quality is significant, albeit insufficiently researched.

Finally, relaxation has a positive impact on sleep quality, as it represents a natural body response that is antagonistic to the stress response. Therefore, relaxation through deliberate interventions like mindful meditation, tai chi and yoga has an opposite effect to stress in that it significantly improves sleep quality. However, more research is required to explore the various relationships between sleep quality and emotive states. The current body of work is limited due to an over-reliance on subjective 
measures of sleep, which may not be indicative of the actual quality and amount of sleep.

\section{ACKNOWLEDGMENTS}

Through the research of this paper, I have gained a deeper understanding of sleep quality and a clearer positioning and interest in psychology.

I would like to show my gratitude to my teachers and educators at the university, who have provided invaluable insight and guidance at every stage of this research.

Further, I would wish to thank all my friends and colleagues for their support. I also thank my family, without whom, I could not have completed this research.

\section{REFERENCES}

[1] Fortier-Brochu, Émilie et al. Relations Between Sleep, Fatigue, And Health-Related Quality Of Life In Individuals With Insomnia. Journal Of Psychosomatic Research, vol 69, no. 5, 2010, pp. 475-483. doi:10.1016/j.jpsychores.2010.05.005.

[2] Kim, Eui-Joong, and Joel E. Dimsdale. "The Effect Of Psychosocial Stress On Sleep: A Review Of Polysomnographic Evidence". Behavioral Sleep Medicine, vol 5, no. 4, 2007, pp. 256-278. doi:10.1080/15402000701557383.

[3] Krystal, Andrew D., and Jack D. Edinger. "Measuring sleep quality." Sleep medicine vol 9, 2008, pp. 10-17. doi: 10.1016/S1389-9457(08)70011-X

[4] Maddock, Clementine, and Carmine M. Pariante. "How Does Stress Affect You? An Overview Of Stress, Immunity, Depression And Disease". Epidemiologia E Psichiatria Sociale, vol 10, no. 3, 2001, pp. 153-162. doi: $10.1017 / \mathrm{s} 1121189 \times 00005285$.

[5] Mirjat, Ali Asghar et al. "Factors Influencing Sleep Quality And Effects Of Sleep On Hypertension". Sleep And Vigilance, vol 4, no. 2, 2020, pp. 125-136. doi:10.1007/s41782-020-00094-5.

[6] National Sleep Foundation. "2011 Poll, Technology And Sleep". Sleep Foundation, 2012, https://www.sleepfoundation.org/professionals/sleepamericar-polls/2011-poll-technology-and-sleep.

[7] Ohayon, Maurice et al. "National Sleep Foundation's Sleep Quality Recommendations: First Report". Sleep Health, vol 3, no. 1, 2017, pp. 6-19. doi:10.1016/j.sleh.2016.11.006.
[8] Pergola, Brianna L. et al. "Sleep Duration Associated with Cardiovascular Conditions Among Adult Nevadans". Sleep Medicine, vol 34, 2017, pp. 209216. doi:10.1016/j.sleep.2017.03.006.

[9] Rusch, Heather L., et al. "The effect of mindfulness meditation on sleep quality: a systematic review and meta-analysis of randomized controlled trials." Annals of the New York Academy of Sciences, vol 1445, no. 1, 2019, pp. 5.

[10] Van Laethem, Michelle, et al. "Bidirectional relations between work-related stress, sleep quality and perseverative cognition." Journal of psychosomatic research, vol 79, no. 5, 2015, pp.391-398.

[11] Zhao, Xiaolin et al. "Perceived Stress And Sleep Quality Among The Non-Diseased General Public In China During The 2019 Coronavirus Disease: A Moderated Mediation Model". Sleep Medicine, vol 77, 2021, $\quad$ pp. 339-345. doi:10.1016/j.sleep.2020.05.021.

[12] Rebar, A. L., Alfrey, K. L., Gardner, B., \& Vandelanotte, C. (2018). Health behaviours of Australian fly-in, fly-out workers and partners during on-shift and off-shift days: An ecological momentary assessment study. BMJ open, 8(12), e023631. 